\section{CASE OF PERMANENT OCCLUSION OF THE UTERUS OBSTRUCTING DELIVERY.}

Bx F. W. FOGARTY, M.D., M.R.C.S., London.

MIRs. $\mathrm{D}-$, aged twenty-five, four years married, previons good health; aborted three months after; successfully operated on for fistula in ano, produced by abscess in perinæum fifteen months subsequent, and recovered rapidly; health in every respect quite re-established; became pregnant Nov., 1848; health continued good; no vaginal discharge, or uterine pains, during the first eight months; was then threatened with premature labour; pains strong and forcing for six hours; no vaginal discharge. Got an opiate; remained well for a week, when sho was again threatened, and treated in a similar way.

On the 10th of August her pains returned, and continued, becoming hourly stronger. No vaginal discharge; bowels open; urine abundant; presentation cannot be reached by the finger.

11th.- Slept little during the night; pains regular and forcing; is uneasy and fretful; bowels active; urine plenty; pulse, 80 . The head has partly entered the pelvis; the os cannot be found; the finger cannot pass in front of the pubis; part of the hand now introduced, and the fingers passed in every direction, but cannot pass the brim of the pelvis, being stopped by the uterine and raginal attachments all round the head, which clearly proves that the head had not escaped from the uterus; a minute examination is now made, but no os uteri can be found. Being now quite satisfied that I had to contend with a case of occlusion from ulceration, or some such canse, I determined to let matters go on qnietly till the head advanced, and that portion of the uterus covering it was more stretched, when I purposed liberating the head by dividing the resisting parts.

12th.-Passed a restless night. Was visited early in the morning by Mr. Alridge, who politely attended in my absence. Pains regular and forcing; bowels open; urine plenty; pulse $86 ;$ complains of want of rest, and exhaustion; head more advanced. Ordered thirty drops of laudanum, and to be again visited at two o'clock P.M., when I purpose operating.-Two o'clock: Is anxious to be delivered; slept a little; pulse 86 ; in other respects, as last. I now examined the presentation through the speculum, in hopes of seeing where the os was originally situated; a glistening, irregular, pearly patch existed where the os might have been expected, but not sufficiently marked to prove it to be a cicatrix, but have no doubt that such it was. I now passed on my finger a sharp-pointed, guarded bistoury, and cut from behind forward to the extent of one inch through the glistening expansion, through which the membranes immediately protruded, and having passed my finger through this opening, as also did Mr. Alridge, and satisfied ourselves that the existing state of things were such as we diagnosed, I continued the incision, by passing the bistoury on my finger between the head and the uterus to the extent of from three to four inches, then withdrawing the finger, and placing it ontside the uterus over the point of the bistoury, passed the point through to the finger, and cut out, as in fistula in ano; having done the same to a less extent in front, the case was left to Nature, and though the pelvis was rather contracted, labour terminated without further assist ance in three hours, by the birth of a full-sized living boy since which time the mother has gone on uniformly well, not having a single bad symptom; she is now up, and able to be about the house.

In such a case as the above it is just possible to mistake it for a head-presentation, where the os was fully dilated and the head passed through. I need scarcely allude to the consequences such a mistake would lead to if it were deemed requisite, from contracted pelvis or other cause, to have recourse to instrumental delivery. It is well also to bear in mind, that in consequence of the stretched state of the uterus the knife should not be carried too far back or forward, so as to avoid wounding the uterine attachments, which must also be extended to a certain extent, and therefore likely to be wounded if this precaution were not taken. At the same time, I think the incision should be as large as these circumstances will admit, so as to prevent a rent which, if once commenced, no knowing where it might end. On the same principle $I$ prefer an extensive longitudinal incision to a crucial one. In the after treatment I was naturally impressed with the importance of preserving a free opening from the uterus, but had no cause to interfere, as the parts healed by granulation, leaving a sufficiently large opening to admit the index-finger.

Percy-street, Pentonville, 1850 .

\section{VOMITING OF BLOOD IN AN INFANT.}

BY W. NIX, Esq., Mile-End Road.

ON Saturday evening, Jan. 19th, a child, thirteen months old was brought to me by its parents, in a state of great exhaustion. They stated, after a slight cough it threw up a large quantity of blood through the mouth and nose; it remained much in the same state, without throwing up more blood, for nearly two hours, and then died. On the following Friday, a post-mortem examination of the body was made by Mr. Snow and myself.

In the chest, the left lung was congested, the heart flaccid, and a more than usual quantity of fluid in the pericardium. In the abdomen, the stomach was found to be of a dark, livit hue, the large intestines and spleen were red; the other viscera healthy. The child was in a general good condition.

Under the above circumstances we deemed it necessary remove the diseased parts, and submit them to Dr. Letheby for analysis. On opening the stomach, it was found to contain about three or four ounces of dark, grumous blood: upon carefully washing this out, the villous cont was found dissolved and in the remaining coa's there were several spots of ccchymosis. The analysis shored slight traces of mercury and oxalic acid, which could be accounted for, but nothing to explain the cause of the great disorganization that had taken place. The above case appcars to be one standing almost by itself, if arising from natural causes. Dr. West, in his Lectures upon Diseases of Children, sars, "Ainong those diseases too seldom met with for any one person to have what can be called real experience about them, may be mentioned vomiting of blood, occasionally observed in infants and young chiidren." (See Medical Gazette, 1818, vol.ii. p. 1016.)

Savile-row, Mile-End Road, Feb. $1 £ 50$.

\section{REPORT OF A CASE OF ACUTE LARYNGITIS.}

BY W. M. FAIRBROTHER, M.D., Kennington.

ON the 28th of Jannary, at ten P.M., I was summoned to see a boy, about cleven years old, who was reported to be alarmingly ill. He had been seen by a neighbouring practitioner in the afternoon, who ordered him some medicine; and on entering the room, I found him half asleep, and drawing his breath with great difficulty, the characteristic sound which accompanies a narrowing of the rima-glottidis being evident at each inspiration, and this had occurred suddenly, when frst attacked, but in a less degree. There was much fever and a rapid pulse; and the respiration was quicker, the obstruction more evident, and the distress greater, upon raising the patient into the sitting posture. These serious symptoms being present, and his voice nearly lost, he was immediately bled, to the extent of twenty-four ounces, before any feeling of faintness was induced. Three grains of calomel, with a small portion of tartar-emetic and Dorer's powder, were given every two hours, and a saline mixture. His bowels acted freely directly after the bloodletting, and he was left for the right.

Jan. 29th.-The patient a little relieved; the countenance somewhat depressed; the laryngeal noises not so frequent nor loud. Ordered the same quantity of calomel, with the Dover's powder, and the tartar-emetic in a mixture. Six leeches over the thyroid cartilage. In the evening, still better; and on the 30th, improving rapidly. The calomel continued, in smaller doses, and at longer intervals. On the 1st of February a trifling hoarseness only remained.

This attack arose from exposure to wet, and is not recorded as presenting anything particularly remarkable, but merely to show that active treatment is the only means of arresting this frightful disease, and that even this is not often successful, unless it is early resorted to.

The employment of blisters, in the early stage, is bad, however useful they may be, if chronic mischief remains. But above all, let not inflammation be mistaken for spasm. It would not have been well for this poor lad, had he taken, as advised, a basin of gruel, with gin added to it, and been left, unaided, till the morning.

Kennington, 1850.

Seamen's Hospital. From the Annual Report of this excellent Institution it appears, that during the past year the number of patients received on board the "Dreadnought" amounted to 2239 , making a total of those remaining from the previous year of 2120 ; of which number 1475 were discharged cured; convalescent, 230; relieved, 54; died, 241; remaining under treatment, 179. The income for the year amounted to $£ 3264$. 